\title{
Opportunities for Electron Backscattered Diffraction Enabled by Direct Electron Detection
}

Fulin Wang ${ }^{1}$, McLean Echlin ${ }^{1}$, Jungho Shin ${ }^{2}$, Benjamin Bammes ${ }^{3}$, Marc De Graef ${ }^{4}$, Tresa Pollock ${ }^{1}$ and Daniel Gianola ${ }^{2}$

${ }^{1}$ University of California-Santa Barbara, Santa Barbara, California, United States, ${ }^{2}$ Materials Department, UC Santa Barbara, Santa Barbara, California, United States, ${ }^{3}$ Direct Electron, LP, San Diego, California, United States, ${ }^{4}$ Carnegie Mellon University, Pittsburgh, Pennsylvania, United States

Electron backscattered diffraction (EBSD) in the scanning electron microscope (SEM) has evolved to be an indispensable technique in materials research for phase identification, crystal orientation determination, orientation gradients, and lattice strain mapping. While tremendous gains in the analysis and indexing of EBSD patterns have occurred, additional promise in advancing new capabilities for EBSD lies in profiting from the collection of EBSD patterns using high performance detectors. Advanced applications of EBSD, such as 3D serial sectioning and in situ testing (e.g. mechanical loading, thermal excursions), demand high speed pattern acquisition while preserving the pattern quality for indexing. In parallel, the ability to discriminate the energy distribution of the exit electrons from the specimen and the energy distribution on the detector is desirable for understanding the physics of electron diffraction, improving pattern quality to extract meaningful features, and even the potential application of EBSD as a spectroscopy tool.

The emergence of modern direct electron detectors (DEDs) has led to breakthroughs in transmission electron microscopy, as well as in EBSD [1,2]. The ultra-high sensitivity of these detectors provides much improved pattern quality, and allows the detection of higher order electron diffraction features (Figure 1). This sensitivity also promises low voltage and/or low current EBSD. Low voltages lead to smaller interaction volume in the material and therefore better spatial resolution. Low currents are amenable to orientation mapping in dose sensitive or non-conductive materials (e.g. geological samples, ceramics). Here, we report the use of a modern DED that is designed and optimized for an SEM environment operating with primary beam energies between 3 and $30 \mathrm{kV}$. Specifically, we evaluate the strengths of electron counting in EBSD, a method that is already widely used in TEM.

For electron counting applications, the detector is set to render a readout rate of 281 frames/second at 2048 by 2048 pixels camera resolution, with a pixel size of $13 \mu \mathrm{m}$. The fast readout speed and high sensitivity of the detector enables the counting of individual electron and thereafter accurate calibration of the pixel intensity values to electron energies. Energy filtering will be demonstrated for a dataset collected at low currents, where individual electrons are both counted and characterized based on their energy. The electron counting events are preserved in a series of fast detector frame readouts (Figure 2), in contrast to signal thresholding on the chip [2] or beam filtering in front of the detector [3]. This additional dimension of data provides access to myriad aspects of the electron signal, e.g. the energy distribution on the detector.

Furthermore, the pixelated detector and fast readout speed allow the use of innovative sampling schemes for the collection of EBSD patterns. For high speed mapping applications, the detector can collect from hundreds of user defined pixel rows at a rate of $>4000$ frames per second. The efficient sampling of

selected parts of the EBSD pattern coupled with dictionary indexing using EMsoft [4, 5] or spherical indexing with EMSphInx [6] can give rise to ultra-fast EBSD orientation mapping [7]. 

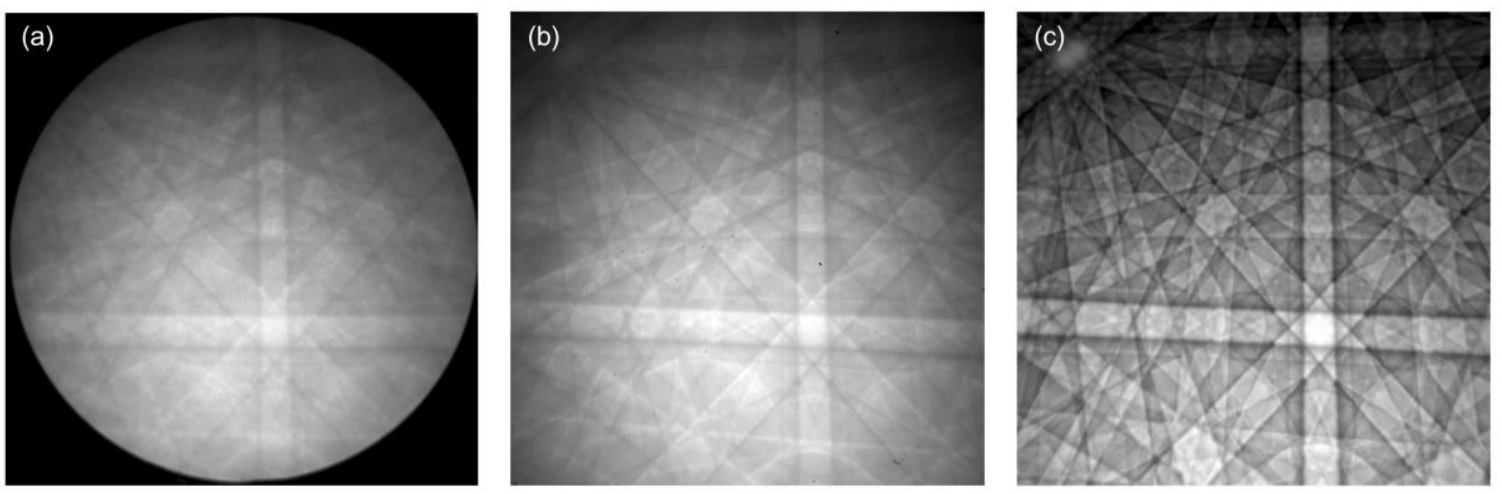

Figure 1. EBSD patterns of single crystal Si acquired at beam voltage $12 \mathrm{kV}$, current $13 \mathrm{nA}$, exposure time $1 \mathrm{~s}$ on (a) conventional phosphor + CCD camera, (b) DED, and (c) simulated by EMsoft. (a) and(b) are raw experimental patterns without background subtraction. Both are adjusted to the same contrast nge.
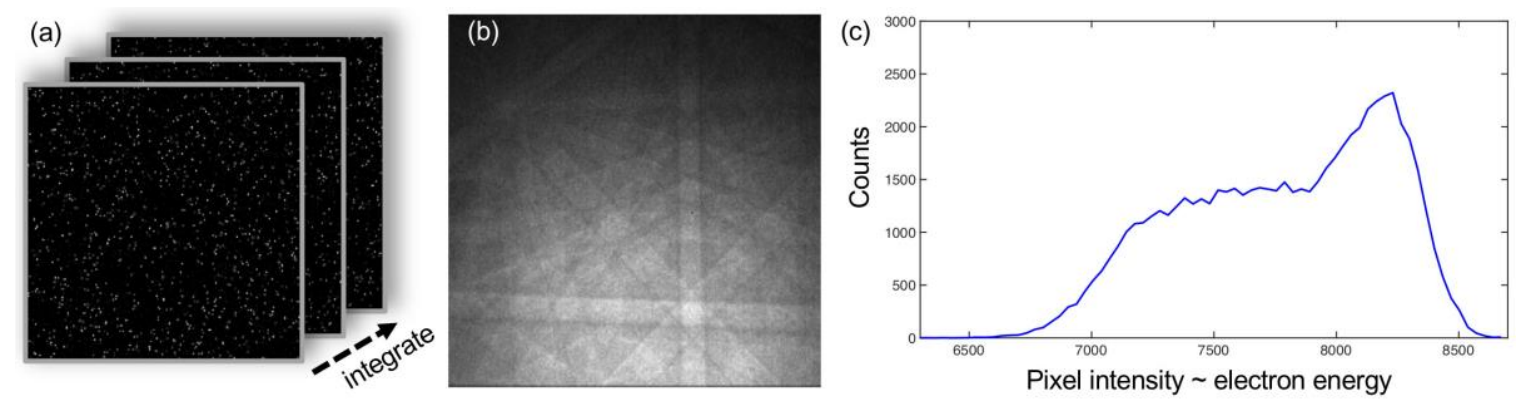

Figure 2. The principle of energy filtered EBSD pattern based on electron counting. The data is acquired on single crystal Si at beam voltage $12 \mathrm{kV}$. The lowest current of $0.78 \mathrm{pA}$ is used. The exposure time is $60 \mathrm{~s}$ in order to obtain significant statistics of electron counting. (a) Schematic showing the integration of fast detector frame readouts (16860 frames in total). (b) Integrated and energy filtered EBSD map. (c) Distribution of pixel intensities in the pattern in (b), the pixel intensity is proportional to the electron energy.

\section{References}

[1] A. Wilkinson, G. Moldovan, T. Britton, A. Bewick, R. Clough and A. Kirkland, Physics Review Letter 111 (2013) p. 065506.

[2] S. Vespucci, A. Winkelmann, G. Naresh-Kumar, K. Mingard, D. Maneuski, P. Edwards, A. Day, V. O'Shea and C. Trager-Cowan, Physics Review B 92 (2015) p. 205301.

[3] A. Deal, T. Hooghan and A. Eades, Ultramicroscopy 108 (2008) p. 116.

[4] P. Callahan and M. De Graef, Microscopy and Microanalysis 19 (2013) p. 1255.

[5] S. Singh, F. Ram and M. De Graef, Microscopy and Microanalysis 23 (2017) p. 212.

[6] W.C. Lenthe, S. Singh, and M. De Graef, Ultramicroscopy 207 (2019) p.112841.

[7] We acknowledge the financial support from the NSF MRSEC Program through DMR 1720256 (IRG-

1). This material is based upon work supported by the U.S. Department of Energy, Office of Basic Energy Sciences, under Award Number DE-SC0019681. The research reported here made use of shared facilities of the UCSB MRSEC (NSF DMR 1720256), a member of the Materials Research Facilities Network (www.mrfn.org).Network (www.mrfn.org). 